\title{
RBEL1 is required for osteosarcoma cell proliferation via inhibiting retinoblastoma 1
}

\author{
HONGHUI TANG* ${ }^{*}$,FENG JI*, JIN SUN, YUE XIE, YONGYI XU and HAITAO YUE \\ Department of Orthopedics, Huai'an First People's Hospital, Nanjing Medical University, Huai'an, Jiangsu 223300, P.R. China
}

Received January 21, 2015; Accepted November 6, 2015

DOI: $10.3892 / \mathrm{mmr} .2015 .4670$

\begin{abstract}
Osteosarcoma is the most common type of primary malignant tumor of the bone. However, mechanisms underlying osteosarcoma cell proliferation are poorly understood. The present study shows that RBEL1, a newly identified Rab-like GTPase, may be a key regulator of osteosarcoma cell proliferation. Knockdown of RBEL1 in osteosarcoma cells resulted in impaired colony formation and cell proliferation. Cell cycle analysis suggested that RBEL1 depletion induced G1-S arrest in osteosarcoma cells. Furthermore, it was demonstrated that retinoblastoma $1(\mathrm{Rb})$ was upregulated and activated following RBEL1 knockdown. In addition, Rb inhibitory downstream targets, such as cyclin A2, cyclin D1, c-Myc and cyclin-dependent kinase 2, were downregulated. $\mathrm{Rb}$ knockdown reversed RBEL1 depletion-induced tumor suppressive effects. In conclusion, the present results suggest that RBEL1 modulates cell proliferation and G1-S transition by inhibiting $\mathrm{Rb}$ in osteosarcoma. These results suggest a potential therapeutic target in osteosarcoma.
\end{abstract}

\section{Introduction}

Osteosarcoma is the most common type of malignant disease in the bone and the third most common malignant tumor in children (1). Although osteosarcoma has a low incidence rate ( $<10 \%$ of all tumors) (2), the disease exhibits aggressive malignant phenotypes and is associated with a high mortality rate (3). The most common location of osteosarcoma development is in the long bones of the limbs (3). Current treatment for osteosarcoma is surgery combined with chemotherapy. However, the 5-year survival rate for patients with metastasis remains $<20 \%(4,5)$. Although intensive efforts have been

Correspondence to: Dr Jin Sun, Department of Orthopedics, Huai'an First People's Hospital, Nanjing Medical University, 6 Beijing Road West, Huai'an, Jiangsu 223300, P.R. China

E-mail: habest126@126.com

*Contributed equally

Key words: RBEL1, osteosarcoma, cell cycle, retinoblastoma 1, $\mathrm{E} 2 \mathrm{~F}$ made, little is known regarding the molecular mechanisms underling this disease.

The RAS superfamily comprises a large number of low-molecular-weight GTP-binding proteins (6). According to the degree of sequence conservation, the superfamily can be divided into five distinct families, including Ras, Rho, Rab, Sar1/Arf and Ran. Rab proteins constitute the largest subfamily, and are key regulators of membrane trafficking processes in eukaryotic cells (7). RBEL1, also termed RABL6, is a novel Rab-like GTPase of unknown function. Recent studies suggest that RBEL1 proteins are overexpressed in breast cancer cells (8) and are involved in cell growth and survival (9). However, the function of RBEL1 in the regulation of tumorigenesis and development of human osteosarcoma is unclear.

The present study investigated the biological function and underlying molecular mechanism of RBEL1 in osteosarcoma. It was demonstrated that RBEL1 participates in the regulation of osteosarcoma cell proliferation. Downregulation of RBEL1 in osteosarcoma resulted in decreased colony formation and cell proliferation rates. G1-S arrest was also observed in RBEL1-depleted cells. Furthermore, it was shown that RBEL1 knockdown activated retinoblastoma $1(\mathrm{Rb})$ and suppressed E2F transcriptional activity. These findings demonstrate that $\mathrm{RBEL} 1$ is a potential oncogene and a novel $\mathrm{Rb}$ inhibitor in osteosarcoma.

\section{Materials and methods}

Cell culture, small interfering (si)RNA transfection and lentivirus infection. U2-OS cells and SAOS2 cells were purchased from the American Type Culture Collection (Manassas, VA, USA) and maintained in Dulbecco's modified Eagle's medium (DMEM; Gibco; Thermo Fisher Scientific, Inc., Waltham, MA, USA) supplemented with $15 \%$ fetal bovine serum (FBS; Gibco; Thermo Fisher Scientific, Inc.) in a humidified atmosphere with $5 \% \mathrm{CO}_{2}$ at $37^{\circ} \mathrm{C}$. For siRNA transfection, siRNA targeting Rb (Cell Signaling Technology Inc., Danvers, MA, USA) and Lipofectamine 2000 (Invitrogen; Thermo Fisher Scientific Inc.) were mixed and incubated for $20 \mathrm{~min}$ at room temperature. Then the mixture was added to cells plated in growth medium without antibiotics. The medium was changed after 4-6 h. For lentivirus infection, lentivirus targeting two different sequences was purchased from Shanghai GenePharma Co., Ltd. (Shanghai, China): KD1 targeting CGG 
CCTAAAGTACCTTCATAA and KD2 targeting CGGCCT AAAGTACCTTCATAA. Cells were plated in growth medium without antibiotics. At the time of infection, growth medium was replaced by medium containing lentivirus and polybrene $(8 \mu \mathrm{g} / \mathrm{ml}$; Santa Cruz Biotechnology, Inc., Santa Cruz, CA, USA) and incubated for $12 \mathrm{~h}$ at $37^{\circ} \mathrm{C}$.

Colony formation assay. Pretreated U2-OS cells and SAOS2 cells infected with shRBEL1-1, shRBEL-2 or scramble lentivirus were plated in 6-well plates (300 cells/well) and were maintained in DMEM supplemented with $15 \%$ FBS in a humidified atmosphere of $5 \% \mathrm{CO}_{2}$ at $37^{\circ} \mathrm{C}$. Growth medium was changed every two days. After 12 days, cultured cells were fixed in 4\% paraformaldehyde (Santa Cruz Biotechnology, Inc.) for $2 \mathrm{~h}$ and stained with $0.1 \%$ crystal violet (Beijing Solarbio Science \& Technology Co., Ltd., Beijing, China) for $20 \mathrm{~min}$. Colonies containing more than 50 individual cells were then counted with the inverted microscope (CKX41; Olympus Corporation, Beijing, China).

Bromodeoxyuridine (BrdU) incorporation assay. Cell proliferation was determined using a Cell Proliferation ELISA kit (containing BrdU labeling solution, FixDenat and anti-BrdU-POD; Roche Diagnostics GmbH, Mannheim, Germany). Briefly, pretreated U2-OS and SAOS2 cells were plated in a 96-well plate. The following day, $10 \mu \mathrm{l} /$ well BrdU labeling solution was added and cells were incubated for $3 \mathrm{~h}$. The medium was replaced by $200 \mu \mathrm{l} /$ well FixDenat and incubated for $30 \mathrm{~min}$ at room temperature. Then FixDenat was removed using $100 \mu \mathrm{l} /$ well anti-BrdU-POD. After washing twice with washing buffer, $100 \mu \mathrm{l} /$ well substrate solution was added. The absorbance was measured at $450 \mathrm{~nm}$ with the ELx808 Absorbance Reader (Bio-Tek Instruments, Inc., Winooski, VT, USA).

Cell cycle analysis. Pretreated U2-OS and SAOS2 cells were harvested and washed twice in phosphate-buffered saline (PBS). Then cells were fixed in $70 \%$ ethanol at $-20^{\circ} \mathrm{C}$ for $4 \mathrm{~h}$. Cells were washed twice with PBS and stained with propidium iodide (PI)/RNase Staining Buffer (BD Biosciences, Franklin Lakes, NJ, USA) for $15 \mathrm{~min}$. Cell cycle distribution was then analyzed by flow cytometry with the FACSCalibur flow cytometer (BD Biosciences).

$R N A$ extraction, $c D N A$ synthesis and reverse transcription-quantitative polymerase chain reaction $(R T-q P C R)$. Total RNA was extracted using TRIzol reagent (Invitrogen, Thermo Fisher Scientific, Inc.) according to the manufacturer's instructions. Reverse transcription was performed using RevertAid First Strand cDNA Synthesis kit (Thermo Fisher Scientific, Inc.) according to the manufacturer's instructions. Real-time PCRs were conducted using an ABI Prism 7500 Detection system (Applied Biosystems, Foster City, CA, USA) and SYBR Select Master mix system (Applied Biosystems) according to the manufacturer's instructions. Briefly, $10 \mu 12 \mathrm{X}$ SYBR Select Master Mix was mixed with primers (200 nM), cDNA template (100 ng) and RNase free water made up to $20 \mu \mathrm{l}$. The cycling conditions werw as follows: $50^{\circ} \mathrm{C}$ for $2 \mathrm{~min}$ and $95^{\circ} \mathrm{C}$ for $2 \mathrm{~min}$, followed by 40 cycles of $95^{\circ} \mathrm{C}$ for $15 \mathrm{sec}$ and $60^{\circ} \mathrm{C}$ for $1 \mathrm{~min}$.
Immunoblot assay. Whole-cell extracts were prepared in radioimmunoprecipitation assay buffer supplemented with $1 \mathrm{mM}$ phenylmethylsulfonyl fluoride (Beijing Solarbio Science \& Technology Co., Ltd.). Total protein concentration was resolved by $10 \%$ SDS-PAGE (Beijing Solarbio Science \& Technology Co., Ltd.). The proteins were then transfered to polyvinylidene difluoride membranes (EMD Millipore, Billerica, MA, USA). Immunoblot analysis was conducted with incubation overnight at $4^{\circ} \mathrm{C}$ with the following antibodies: Anti-RBEL1 (1:1,000; ab111866) from Abcam (Cambridge, MA, USA), anti-Rb (1:200; 554144) and anti-active $\mathrm{Rb}(1: 200 ; 554164)$ from (BD Biosciences), anti-c-Myc (1:100; sc-789), anti-cyclin D1 (1:200; sc-25765), anti-cyclin A2 (1:200; sc-53234) and anti-cyclin-dependent kinase 2 (CDK2; 1:200; sc-748) from Santa Cruz Biotechnology Inc., and polyclonal rabbit anti-human tubulin $(1: 2,000 ;$ T2200) from (Sigma-Aldrich, St. Louis, MO, USA). Membranes were then incubated with horseradish peroxidase (HRP)-conjugated anti-rabbit immunoglobulin $\mathrm{G}$ secondary antibodies (1:5,000; ZB-2301; Beijing Zhongshan Golden Bridge Biotechnology Co., Ltd., Beijing, China) and was detected using the Western Chemiluminescent HRP Substrate (EMD Millipore).

Statistical analysis. All data sets were analyzed by Student's t-test. Data are presented as the mean \pm standard deviation from three independent experiments. Statistical analysis was conducted using Graphpad Prism software, version 6 (GraphPad Software, Inc., San Diego, CA, USA). P $<0.05$ was considered to indicate a statistically significant difference.

\section{Results}

RBEL1 depletion in osteosarcoma cells suppresses colony formation and cell proliferation. To explore the potential role of RBEL1 in osteosarcoma, its expression in U2-OS and SAOS2 osteosarcoma cell lines was inhibited by shRNA lentivirus targeting two different sequences. Knockdown efficiency was confirmed by RT-qPCR and immunoblot analysis (Fig. 1A and B). Then RBEL1 knockdown cells and negative control cells were subjected to a colony formation assay. As shown in Fig. 1C, colony number and size were suppressed following RBEL1 knockdown. Furthermore, a BrdU incorporation assay was performed to determine whether RBEL1 depletion also had an impact on cell proliferation. In addtion, in U2-OS and SAOS2 cells RBEL1 depletion significantly inhibited BrdU incorporation suggesting suppressed proliferation (Fig. 1D). These data suggest that RBEL1 may act as a tumor suppressor in osteosarcoma.

RBEL1 depletion suppresses G1-S transition in osteosarcoma cells. The cell cycle of eukaryotic cells is conventionally divided into four phases and requires three switch-like transitions at the onset of S phase and at entry and exit of mitosis. Among them, G1-S transition is the most deregulated cell cycle control mechanism in malignant diseases (10). Thus it was hypothesized that RBEL1 may modulate G1-S transition thereby promoting cell proliferation in osteosarcoma cells. Using flow cytometry, cell cycle distribution of U2-OS and SAOS2 cells infected with RBEL1 shRNA or negative control shRNA was examined. As shown in Fig. 2, RBEL1 depletion 

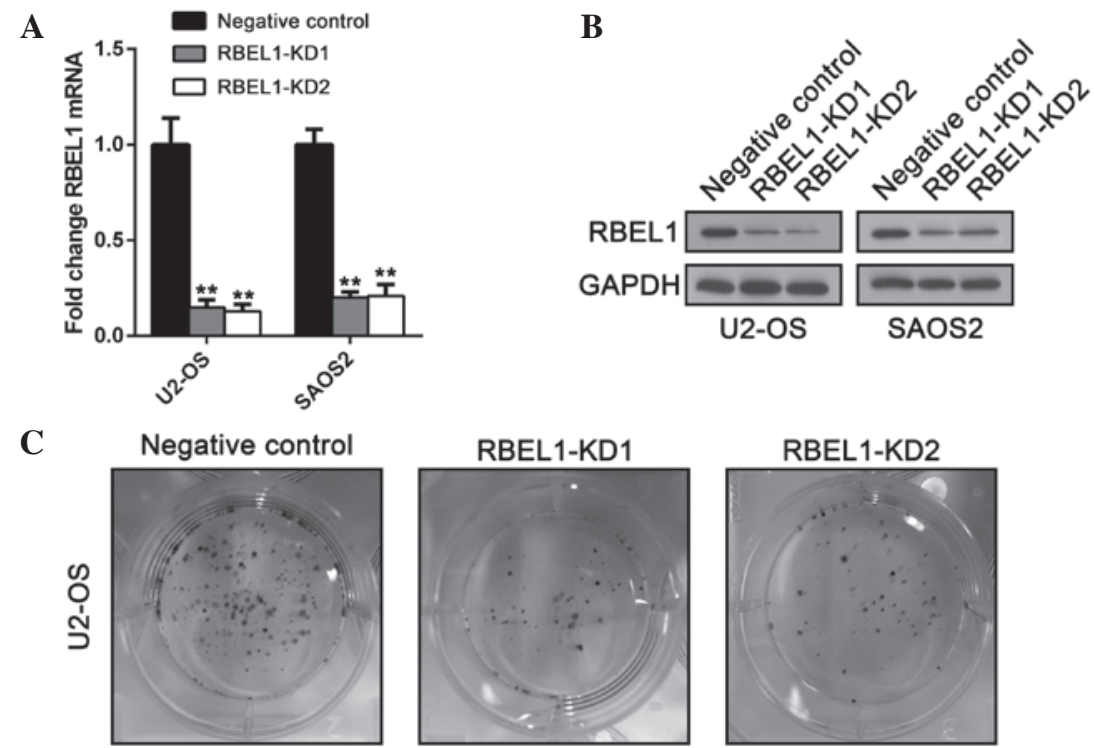

D

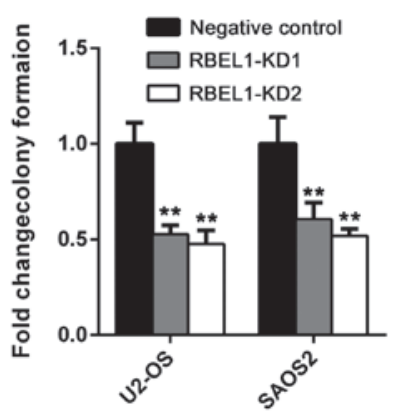

$\mathbf{E}$

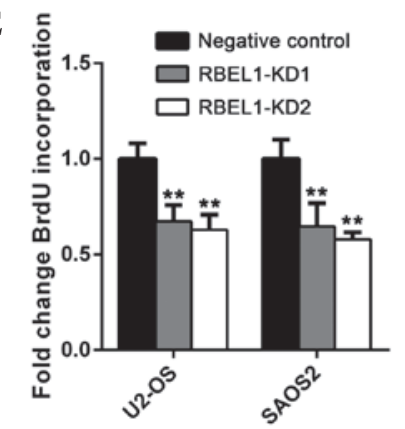

Figure 1. RBEL1 depletion in osteosarcoma cells suppresses colony formation and cell proliferation. U2-OS and SAOS2 cells were infected with RBEL1 shRNA lentivirus (RBEL1-KD1 or -KD2) or negative control lentivirus. After $72 \mathrm{~h}$, cell were harvested and analyzed using (A) reverse transcription-quantitative polymerase chain reaction and (B) immunoblot assay. RBEL1-KD cells or negative control cells were plated in 6-well plates (300 cells/well) and medium was replaced every two days. (C) After 12 days, cells were fixed and stained with crystal violet. (D) Then colony numbers were calculated. (E) Cell proliferation was determined by a BrdU incorporation assay. The data are presented as the mean \pm standard deviation $(\mathrm{n}=3)$. $\mathrm{P}<0.05$ and ${ }^{* *} \mathrm{P}<0.01$, compared with the negative control. shRNA, short hairpin RNA; BrdU, bromodeoxyuridine.

in U2-OS cells resulted in enhanced G1-S arrest compared with negative control cells. Similar results were also shown in SAOS2 cells. These results indicate that RBEL1 may participate in G1-S transition regulation in osteosarcoma.

$R B E L 1$ regulates $R b$ activity in osteosarcoma cells. $\mathrm{Rb}$ is the major player in cell cycle control $(11,12)$. A recent study indicated that RBEL1 negatively regulates $\mathrm{Rb}$ activation (8). Therefore, it was hypothesized that RBEL1 may regulate G1-S transition by inhibiting $\mathrm{Rb}$ activation. The impact of RBEL1 depletion on $\mathrm{Rb}$ expression was analyzed. An immunoblot assay showed that following RBEL1 knockdown, osteosarcoma cells exhibited increased expression of $\mathrm{Rb}$ compared with control cells (Fig. 3A). As the hypophosphorylated form of $\mathrm{Rb}$ is the active form that suppresses cell proliferation, it was examined whether hypophosphorylated $\mathrm{Rb}$ was also upregulated. As expected, RBEL1 knockdown cells expressed more hypophosphorylated Rb compared with the control (Fig. 3A). It is well-known that $\mathrm{Rb}$ targets the E2F transcription factor to suppress its downstream oncogene expression $(11,12)$. To investigate whether RBEL1 depletion affects E2F transciptional activity, the expression of its downstream targets, such as cyclin A2, cyclin D1, c-Myc and CDK2, were examined.
In agreement with above results, the mRNA and protein expression of these oncogenes was downregulated following RBEL1 knockdown in both cell types (Fig. 3B-D). These results indicate that $\mathrm{RBEL1}$ may be a key regulator of $\mathrm{Rb}$ and its downstream targets.

RBEL1 exerts its oncogenic effects by inhibiting $R b$. The results indicated that RBEL1 modulates cell proliferation and G1-S transition, and can inhibit Rb activity. Thus it was investigated whether RBEL1 exerts its oncogenic effects by inhibiting $\mathrm{Rb}$. To test this hypothesis, $\mathrm{Rb}$ expression was suppressed in RBEL1 knockdown cells to examine whether RBEL1 knockdown-induced tumor suppression can be rescued. The knockdown efficiency was confirmed by RT-qPCR and an immunoblot assay (Fig. 4A and B). A flow cytometry assay showed that in U2-OS and SAOS2, RBEL1 knockdown-induced G1-S arrest was decreased after Rb suppression (Fig. 4C and D). Furthermore, Rb suppression reversed colony formation capability in cells with RBEL1-knockdown (Fig. 4E). BrdU incorporation was also increased following $\mathrm{Rb}$ suppression (Fig. 4F). These results suggest that RBEL1 exerts its oncogenic effects by inhibiting $\mathrm{Rb}$. 
A

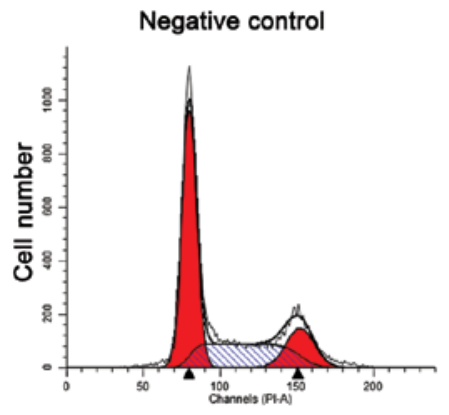

REBEL1-KD1

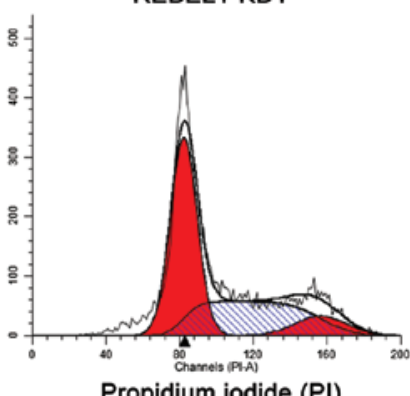

REBEL1-KD2

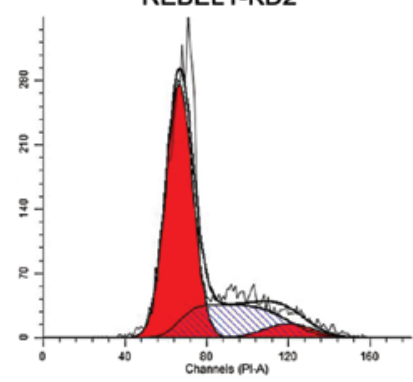

B
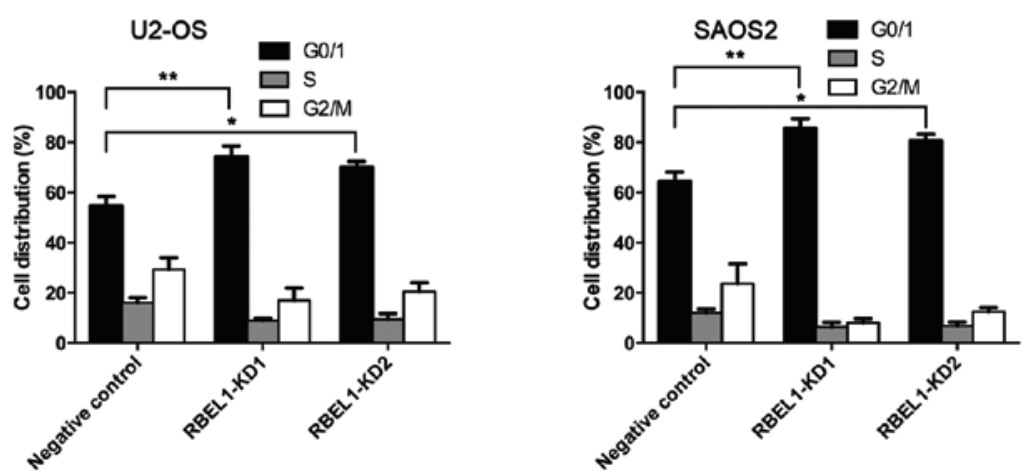

Figure 2. RBEL1 depletion suppresses G1-S transition in osteosarcoma cells. U2-OS and SAOS2 cells were infected with RBEL1 shRNA lentivirus (RBEL1-KD1 or -KD2) or negative control lentivirus. (A) Cell cycle distribution was determined by flow cytometry. (B) Representative histograms of U2-OS cell cycle distribution. The data are presented as the mean \pm standard deviation $(n=3)$. ${ }^{*} \mathrm{P}<0.05$ and $^{* * *} \mathrm{P}<0.01$, compared with the negative control. shRNA, short hairpin RNA.

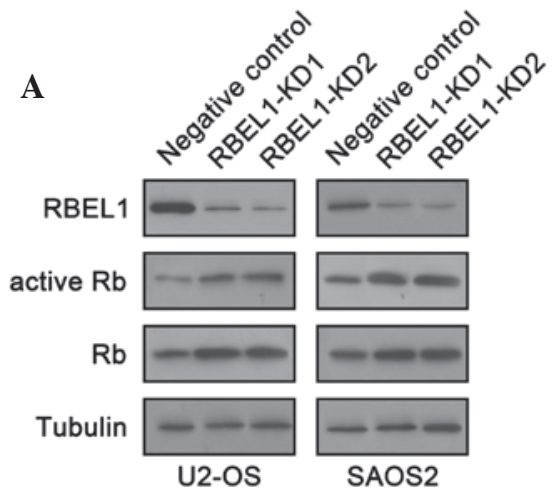

C

D
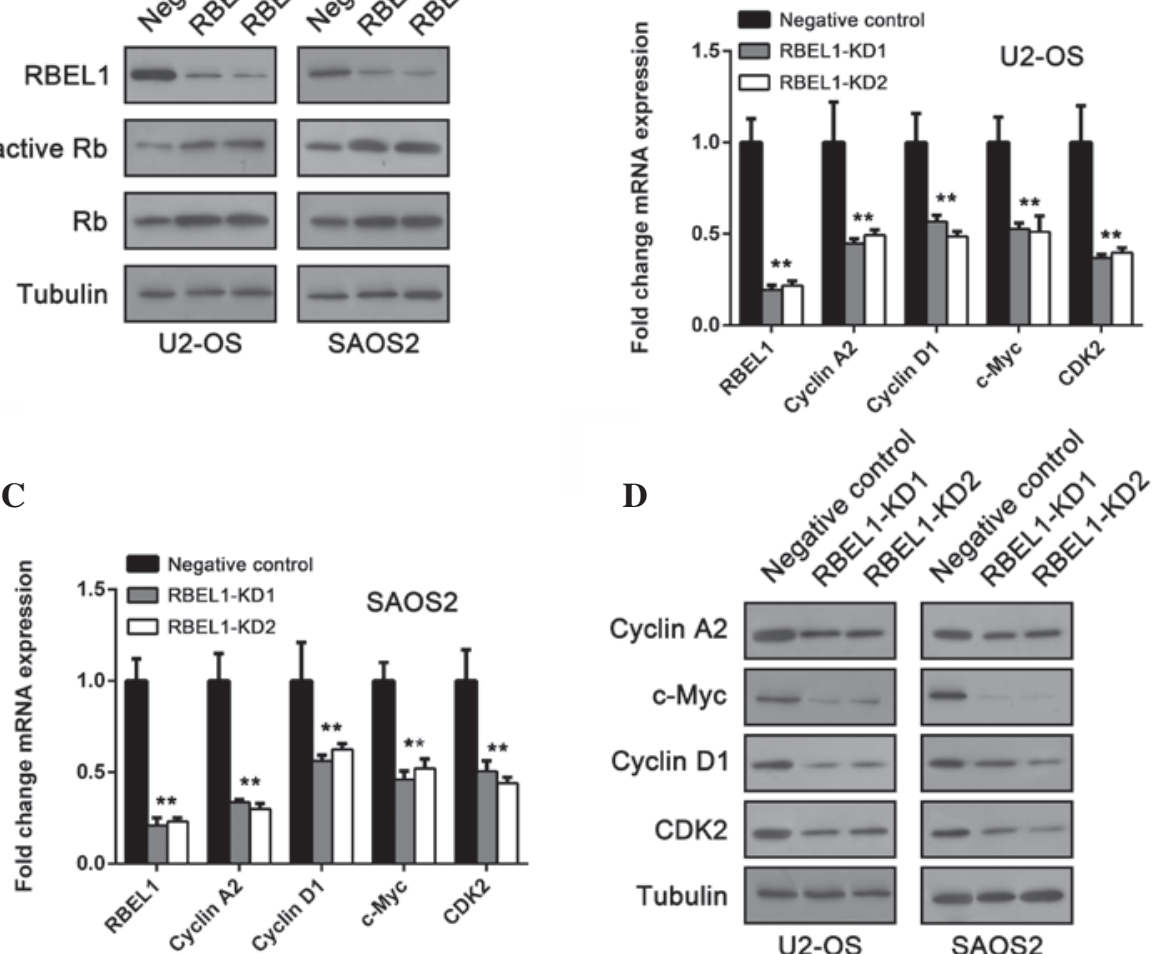

B
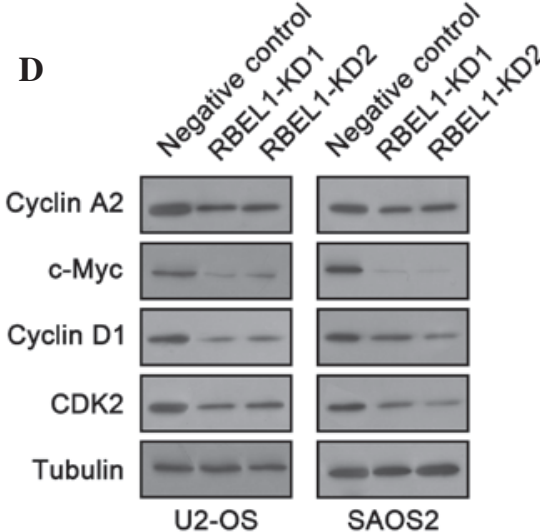

Figure 3. RBEL1 regulates Rb activity in osteosarcoma cells. (A) U2-OS and SAOS2 cells were infected with RBEL1 shRNA lentivirus (RBEL1-KD1 or -KD2) or negative control lentivirus. After $48 \mathrm{~h}$, cells were harvested and subjected to an immunoblot assay with the indicated antibodies. E2F downstream target mRNA expression was analyzed by reverse transcription-quantitative polymerase chain reaction in (B) U2-OS and (C) SAOS2 cells. (D) E2F downstream target protein expression was analyzed by an immunoblot assay in U2-OS and SAOS2 cells. The data are presented as the mean \pm standard deviation $(\mathrm{n}=3) .{ }^{*} \mathrm{P}<0.05$ and ${ }^{* * *} \mathrm{P}<0.01$, compared with negative control. 
A

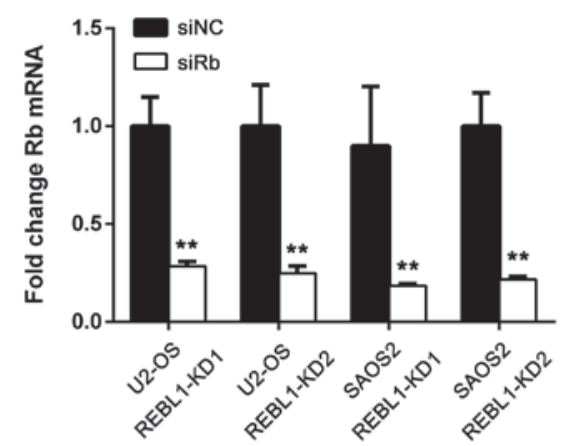

C

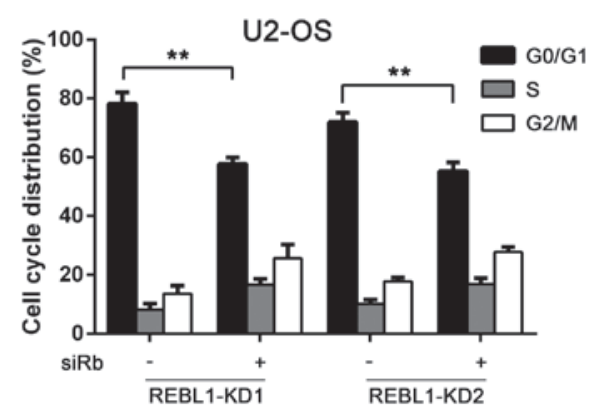

$\mathbf{E}$

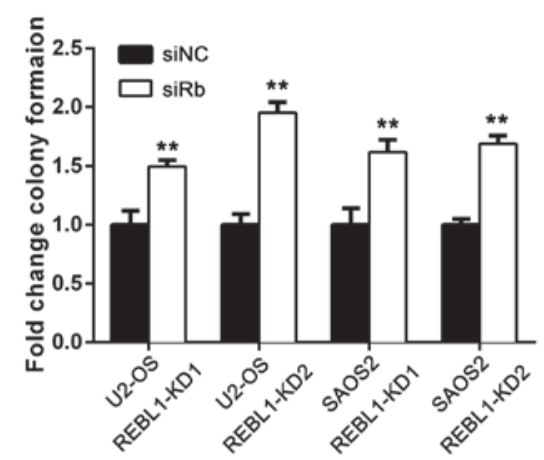

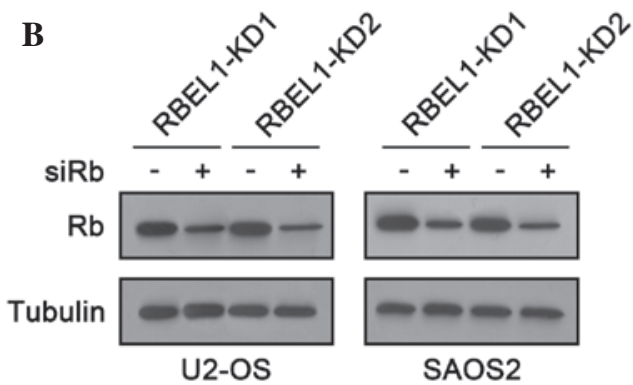

D

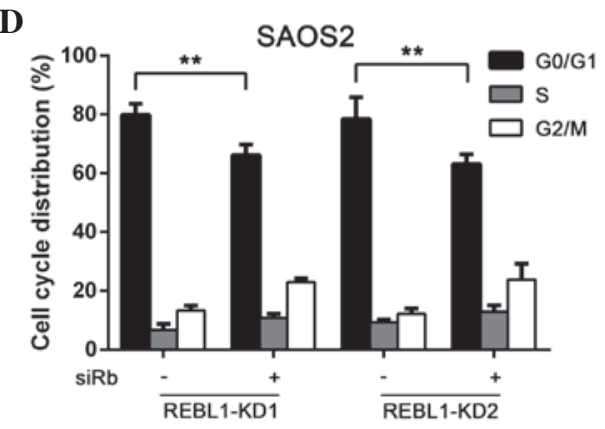

$\mathbf{F}$ 으

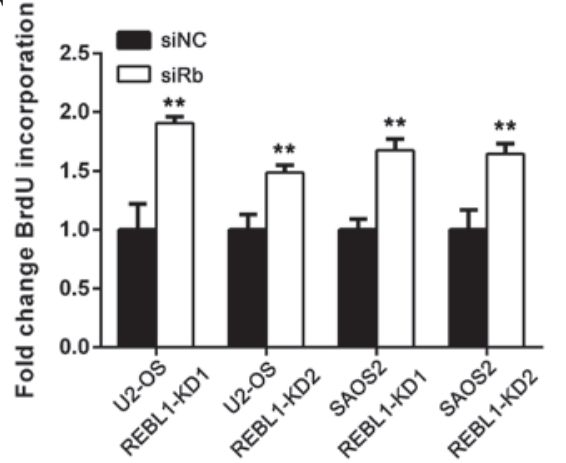

Figure 4. RBEL1 exerts its oncogenic effects by inhibiting Rb. U2-OS and SAOS2 cells infected with RBEL1 shRNA lentivirus were transfected with either siRb or siNC. Then knockdown efficiency was analyzed by (A) reverse transcription-quantitative polymerase chain reaction or (B) immunoblot assay. Cell cycle distribution alteration was analyzed by flow cytometry in (C) U2-OS and (D) SAOS2 cells. A (E) colony formation assay and (F) BrdU incorporation assay were also performed. The data are presented as the mean \pm standard deviation $(\mathrm{n}=3)$. ${ }^{*} \mathrm{P}<0.05$ and ${ }^{* *} \mathrm{P}<0.01$ compared with negative control. siNC, negative control siRNA siRb siRNA targeting Rb; BrdU, bromodeoxyuridine; CDK, cyclin-dependent kinase 2.

\section{Discussion}

Numerous factors have been implicated in tumorigenesis, such as mutations, chronic inflammation resulting from bacterial or viral infection, and prolonged exposure to radiation or oncogenic chemicals $(13,14)$. However, the etiology of osteosarcoma remains largely unknown. Deregulated cell proliferation has been recognized as one of the hallmarks of cancer $(15,16)$. Intensive efforts were made and numerous genetic or epigenetic events were attributed to proliferation deregulation (17-22). The present study demonstrates a novel regulatory mechanism of cell proliferation in osteosarcoma cells. When RBEL1 was depleted, U2-OS and SAOS2 cells generated fewer and smaller colonies. Cell proliferation suppression and G1-S arrest was also observed in RBEL1-depleted cells. These results suggest RBEL1 may act as an oncogene in osteosarcoma.

The cell cycle is a tightly regulated process that ensures specific events take place in an orderly manner. Any fault in this regulatory network results in uncontrolled cell proliferation or cell death $(23,24)$. The cell cycle is monitored by checkpoints that sense possible defects during DNA synthesis and chromosome segregation. Cell cycle arrest allows cells to properly repair these defects, thus preventing their transmission to the resulting daughter cells (25). Deregulated cell cycle arrest has been observed in osteosarcoma $(26,27)$. However, the underlying molecular mechanism remains unclear. In the present study, RBEL1 was observed to participate in cell cycle control in osteosarcoma. G1-S transition is the most common deregulated cell cycle transition in cancer (23) and in the present study RBEL1 downregulation induced G1-S arrest in $\mathrm{U} 2-\mathrm{OS}$ and $\mathrm{SAO} 2$ cells.

$\mathrm{Rb}$ is a tumor suppressor protein that is dysfunctional in several types of cancer $(28,29)$. One function of $\mathrm{Rb}$ is to prevent excessive cell growth by inhibiting cell cycle progression until a cell is ready to divide $(11,30)$. RBEL1 was found to negatively regulate $\mathrm{Rb}$ activity. RBEL1 downregulation resulted in an increase in $\mathrm{Rb}$ expression and an increase in the hypophosphorylated form of the protein. Furthermore, E2F transcriptional activity was also reduced. Cyclin A2, cyclin D1, c-Myc and CDK2, the downstream target of E2F transcription factors, 
were upregulated in RBEL1-depleted cells. These proteins are widely accepted as oncogenes and cell cycle positive regulator in cancer cells. Thus, RBEL1 may promote cell proliferation and G1-S transition by inhibiting $\mathrm{Rb}$ activity.

In conclusion, these findings suggest a novel mechanism underlying osteosarcoma progression and cell cycle regulation. RBEL1 inhibits $\mathrm{Rb}$ activity to upregulated oncogenic factors, such as cyclin A2, cyclin D1, c-Myc and CDK2, therefore promoting G1-S transition and cell proliferation in osteosarcoma cells. These results suggest that RBEL1 may be a novel therapeutic target and potential biomarker for osteosarcoma. However, whether RBEL1 is upregulated in human osteosarcoma samples and how RBEL1 exerts its Rb inhibitory function remain unclear and require further investigation.

\section{References}

1. Moore DD and Luu HH: Osteosarcoma. Cancer Treat Res 162 65-92, 2014.

2. Siegel R, Ma J, Zou Z and Jemal A: Cancer statistics, 2014. CA Cancer J Clin 64: 9-29, 2014.

3. Kansara M, Teng MW, Smyth MJ and Thomas DM: Translational biology of osteosarcoma. Nat Rev Cancer 14: 722-735, 2014.

4. Nakamura T, Matsumine A, Matsubara T, Asamuma K, Niimi R, Uchida A and Sudo A: Retrospective analysis of metastatic sarcoma patients. Oncol Lett 2: 315-318, 2011.

5. Marina N, Gebhardt M, Teot L and Gorlick R: Biology and therapeutic advances for pediatric osteosarcoma. Oncologist 9: 422-441, 2004.

6. Downward J: Targeting RAS signalling pathways in cancer therapy. Nat Rev Cancer 3: 11-22, 2003.

7. Stenmark H: Rab GTPases as coordinators of vesicle traffic. Nat Rev Mol Cell Biol 10: 513-525, 2009.

8. Montalbano J, Jin W, Sheikh MS and Huang Y: RBEL1 is a novel gene that encodes a nucleocytoplasmic Ras superfamily GTP-binding protein and is overexpressed in breast cancer. J Biol Chem 282: 37640-37649, 2007.

9. Montalbano J, Lui K, Sheikh MS and Huang Y: Identification and characterization of RBEL1 subfamily of GTPases in the Ras superfamily involved in cell growth regulation. J Biol Chem 284 18129-18142, 2009.

10. Hochegger H, Takeda S and Hunt T: Cyclin-dependent kinases and cell-cycle transitions: Does one fit all? Nat Rev Mol Cell Biol 9: 910-916, 2008.

11. Burkhart DL and Sage J: Cellular mechanisms of tumour suppression by the retinoblastoma gene. Nat Rev Cancer 8 : 671-682, 2008.
12. Classon $\mathrm{M}$ and Harlow E: The retinoblastoma tumour suppressor in development and cancer. Nat Rev Cancer 2: 910-917, 2002.

13. Travis LB, Demark Wahnefried W, Allan JM, Wood ME and $\mathrm{Ng} \mathrm{AK}$ : Aetiology, genetics and prevention of secondary neoplasms in adult cancer survivors. Nat Rev Clin Oncol 10: 289-301, 2013.

14. Barcellos-Hoff MH: Does microenvironment contribute to the etiology of estrogen receptor-negative breast cancer? Clin Cancer Res 19: 541-548, 2013.

15. Hanahan D and Weinberg RA: The hallmarks of cancer. Cell 100: 57-70, 2000.

16. Hanahan D and Weinberg RA: Hallmarks of cancer: The next generation. Cell 144: 646-674, 2011.

17. Gupta SC, Kim JH, Prasad S and Aggarwal BB: Regulation of survival, proliferation, invasion, angiogenesis and metastasis of tumor cells through modulation of inflammatory pathways by nutraceuticals. Cancer Metastasis Rev 29: 405-434, 2010.

18. Fritz V and Fajas L: Metabolism and proliferation share common regulatory pathways in cancer cells. Oncogene 29: 4369-4377, 2010.

19. Whitfield ML, George LK, Grant GD and Perou CM: Common markers of proliferation. Nat Rev Cancer 6: 99-106, 2006.

20. Hwang HW and Mendell JT: MicroRNAs in cell proliferation, cell death and tumorigenesis. Br J Cancer 94: 776-780, 2006.

21. Deng H, Cheng Y, Guo Z, Zhang F, Lu X, Feng L, Wang X and $\mathrm{Xu} Z$ : Overexpression of Cyclin A2 ameliorates hypoxia-impaired proliferation of cardiomyocytes. Exp Ther Med 8: 1513-1517, 2014.

22. Chen G, Wang H, Xie S, Ma J and Wang G: STAT1 negatively regulates hepatocellular carcinoma cell proliferation. Oncol Rep 29: 2303-2310, 2013.

23. Bertoli C, Skotheim JM and de Bruin RA: Control of cell cycle transcription during G1 and S phases. Nat Rev Mol Cell Biol 14: 518-528, 2013.

24. Tian Y, Wan H and Tan G: Cell cycle-related kinase in carcinogenesis. Oncol Lett 4: 601-606, 2012.

25. Malumbres M and Barbacid M: Cell cycle, CDKs and cancer: A changing paradigm. Nat Rev Cancer 9: 153-166, 2009.

26. Zhang L, Zhou Q, Zhang N, Li W, Ying M, Ding W, Yang B and $\mathrm{He} \mathrm{Q}: \mathrm{E} 2 \mathrm{~F} 1$ impairs all-trans retinoic acid-induced osteogenic differentiation of osteosarcoma via promoting ubiquitination-mediated degradation of RAR $\alpha$. Cell Cycle 13: 1277-1287, 2014

27. Wang XH, Cai P, Wang MH and Wang Z: MicroRNA-25 promotes osteosarcoma cell proliferation by targeting the cell cycle inhibitor p27. Mol Med Rep 10: 855-859, 2014.

28. Dick FA and Rubin SM: Molecular mechanisms underlying RB protein function. Nat Rev Mol Cell Biol 14: 297-306, 2013.

29. Chen HZ, Tsai SY and Leone G: Emerging roles of E2Fs in cancer: An exit from cell cycle control. Nat Rev Cancer 9: 785-797, 2009.

30. Prior IA, Lewis PD and Mattos C: A comprehensive survey of Ras mutations in cancer. Cancer Res 72: 2457-2467, 2012. 